\title{
Efficient Techniques for Multipolynomial Resultant Algorithms
}

\author{
Dinesh Manocha and John Canny \\ Computer Science Division \\ University of California \\ Berkeley, CA 94720
}

\begin{abstract}
Computational methods for manipulating sets of polynomial equations are becoming of greater importance due to the use of polynomial equations in various applications. In some cases we need to eliminate variables from a given system of polynomial equations to obtain a "symbolically smaller" system, while in others we desire to compute the numerical solutions of non-linear polynomial equations. Recently, the techniques of Gröbner bases and polynomial continuation have received much attention as algorithmic methods for these symbolic and numeric applications. When it comes to practice, these methods are slow and not effective for a variety of reasons. In this paper we present efficient techniques for applying multipolynomial resultant algorithms and show their effectiveness for manipulating system of polynomial equations. In particular, we present efficient algorithms for computing the resultant of a system of polynomial equations (whose coefficients may be symbolic variables). These algorithms can be used for interpolating polynomials from their values and expanding symbolic determinants. Moreover, we use multipolynomial resultants for computing the real or complex solutions of non-linear polynomial equations. We also discuss the implementation of these algorithms in the context of certain applications.
\end{abstract}

Permission to copy without fee all or part of this material is granted provided that the copies are not made or distributed for direct commercial advantage, the ACM copyright notice and the title of the publication and its date appear, and notice is given that copying is by permission of the Association for Computing Machinery. To copy otherwise, or to republish, requires a fee and/or specific permission.

(c) 1991 ACM 0-89791-437-6/91/0006/0086 ..\$1.50

\section{Introduction}

Finding the solution to a system of non-linear polynomial equations over a given field is a classical and fundamental problem in computational algebra. This problem arises in symbolic and numeric techniques used to manipulate sets of polynomial equations. Many applications in computer algebra, robotics, geometric and solid modeling use sets of polynomial equations for object representation (as a semialgebraic set) and for defining constraints (as an algebraic set). The main operations in these applications can be classified into two types: simultaneous elimination of one or more variables from a given set of polynomial equations to obtain a "symbolically smaller" system and computing the numeric solutions of a system of polynomial equations. Elimination theory, a branch of classical algebraic geometry, investigates the condition under which sets of polynomials have common roots. Its results were known at least a century ago [Ma16; Sa1885; Wd50] and still appear in modern treatments of algebraic geometry, although in non-constructive form. The main result is the construction of a single resultant polynomial of $n$ homogeneous polynomial equations in $n$ unknowns, such that the vanishing of the resultant is a necessary and sufficient condition for the given system to have a non-trivial solution. We call this resultant the $\mathrm{Mul}$ tipolynomial Resultant ${ }^{1}$ of the given system of polynomial equations. The multipolynomial resultant of the system of polynomial equations can be used for eliminating the variables and computing the numeric solutions of a given system of polynomial equations.

Recently the technique of Gröbner bases has received much attention as an algorithmic method for determining properties of polynomial equations [Bu85; Bu89]. Its applications include ideal membership besides eliminating a set of variables or computing the numerical solutions of a system of polyno-

\footnotetext{
${ }^{1}$ Other authors have used the term multiequational resultants [BGW88]
} 
mial equations. One of the main difficulties in using Grobbner bases is that the method may be slow for even small problems. In the worst case, its running time may can be doubly exponential in the number of variables [MM82]. Even in special cases where this doubly exponential behavior is not observed, deriving tight upper bounds on the method's running time is difficult. This behavior is also observed in practice. For example, it is difficult to implicitize even low degree parametric surfaces by using Gröbner bases in a reasonable amount of time and space [Ho90]. Furthermore, issues of numerical stability make them unattractive for computing numeric solutions of polynomial equations [Mo90].

As far as numerical applications are concerned the technique of polynomial continuation has gained importance for computing the full list of geometrically isolated solutions to a system of polynomial equations. They have been used in many robotics and engineering applications [Mo87; WM90]. Although continuation methods have a solid theoretical background and a high degree of computational reliability (in some cases), its usage is limited to applications requiring all the solutions in the complex domain and thereby making them slow for some practical applications where only real solutions are needed. In many cases the system of polynomial equations may have a high Bezout number (the total number of solutions in the complex domain), but we are only interested in the solutions in a small subset of the real domain (the domain of interest). The continuation technique requires starting with a particular system of equations having the same Bezout number as the given system and marching along to compute all the solutions of the given system. It is difficult to restrict them to computing the solutions in the domain of interest.

Multipolynomial resultant algorithms provide the most efficient methods (as far as asymptotic complexity is concerned) for solving systems of polynomial equations or eliminating variables [BGW88]. Their main advantage lies in the fact that the resultant can always be expressed as the ratio of two determinants and for small values of $n$ (where $n$ is the number of equations), as the determinant of a single matrix. As a result, we are able to use algorithms from linear algebra and obtain tight bounds on the running times of multipolynomial resultant algorithms. Furthermore, in many symbolic and numeric applications, we may choose not to expand the determinants and use properties of matrices and determinants to incorporate the use of resultants in the specific applications [MC90; MC91a].

In this paper we present an interpolation based algorithm to compute the resultant of a system of polynomial equations to obtain a "symbolically smaller" system. The algorithm can be used for interpolating polynomials from their values and expanding symbolic determinants. We also describe an efficient implementation of the algorithm and present its performance for applications like implicitization. Moreover, we effectively use resultants for computing the numeric solutions of a system of polynomial equations in the domain of interest. In this case, we reduce the problem to computing eigenvalues of a matrix. Efficient algorithms for computing eigenvalues are well known and their implementations are available as part of standard packages like EISPACK [GV89]. Furthermore, in the context of floating point computations, the numerical accuracy of these operations is well understood. The rest of the paper is organized as follows. In Section 2 we give a brief preview of different formulations used for computing the resultant of a system of polynomials. In Section 3, we present an algorithm for eliminating one or more variables from the given system of equations and express the resultant as a polynomial in the coefficients of given equations (which may be symbolic variables) and finally in Section 4, we give details of the algorithm used for computing the numeric solutions of a given system of polynomials in the domain of interest.

\section{Multipolynomial Resultants}

Given $\boldsymbol{n}$ homogeneous polynomial in $\boldsymbol{n}$ unknowns, the resultant of the given system of equations is a polynomial in the coefficients of the given equations. The most familiar form of the resultant is the Sylvester's formulation for the case $n=2$. In this case, the resultant can always be expressed as determinant of a matrix. However, a single determinant formulation may not exist for any arbitrary $n$ and the most general formulation of resultant (to the best of our knowledge) expresses it as a ratio of two determinants [Ma02; Ma21]. Many a times both the determinants evaluate to zero. To compute the resultant we need to perturb the equations and use limiting arguments. This corresponds to computing the characteristic polynomials of both the determinants [Ca87]. The ratio of the two characteristic polynomials is termed the Generalized Characteristic Polynomial, and the resultant corresponds to its constant term [Ca88]. If the constant term is zero, its lowest degree term contains important information and can be used for computing the proper components in the presence of excess components. This formulation has advantages for both numeric and symbolic applications [Ca88; MC90]. Many special cases, corresponding to $n=2,3,4,5,6$ when the resultant can be 
expressed as the determinant of a matrix, are given in [Di08; Jo89; Mo25; MC27].

\section{Symbolic Elimination}

In this section we present an algorithm for efficiently computing the resultant of a system of polynomial equations, whose coefficients may be symbolic variables. Computing the resultant involves constructing the corresponding matrices from the given system of equations and evaluating their determinants. The entries of the matrices are polynomial functions of the coefficients of the polynomial equations. As such it should be relatively easy to implement such an algorithm within the framework of a computer algebra system. However, these systems take a lot of time for evaluating even low order symbolic determinants. Consider the problem of implicitizing bicubic parametric surface, whose implicit representation is a degree 18 polynomial in 3 variables, say $x, y$ and $z$. In this case, the resultant of the parametric equations correspond to a $18 \times 18$ determinant and each of its entries is a linear polynomial in $x, y$, and $z$. However, standard computer algebra systems (available on most workstations) are not able to evaluate such determinants in a reasonable amount of time and space [MC90]. Most of the time they run for a long period of time and crash because of their memory limitations.

There are many reasons for the failure and bad performance of symbolic determinant expansion algorithms implemented within the framework of computer algebra systems.

1. Most computer algebra systems use sparse representation for multivariate polynomials and the computations become slow whenever the polynomials generated are dense.

2. The algorithms used are symbolic in nature and perform operations like polynomial addition, multiplication on the input and the intermediate expressions being generated. The arithmetic for these symbolic operations is expensive. For example, the cost of multiplying multivariate polynomials is quadratic for most implementations. Moreover, the algorithm may generate large intermediate expressions. For example when using straight-forward Gaussian elimination over the polynomial entry domain, it can happen that intermediate subdeterminants are very large polynomials while the final answer is an expression of modest size. Furthermore, their implementations in lisp-like environments may require a large amount of virtual memory and thereby slowing down the computations.

3. These systems use exact arithmetic and represent the coefficients of intermediate expressions as bignums. As a result, the cost of arithmetic operations is quadratic in the coefficient size. The coefficient size is proportional to the degree of the intermediate polynomial expressions being generated and tends to grow exponentially with the degree.

The bottleneck in the resultant algorithms is the symbolic expansion of determinants. We therefore, chose not to work within the environment of computer algebra systems and rather use an algorithm based on multivariate interpolation to compute the symbolic determinants. As a result, the resulting algorithm involves numeric computations and no intermediate symbolic expressions are generated. This takes care of the problem of generating large intermediate symbolic expression. However, the magnitude of the intermediate numbers grows and we need to use bignum arithmetic for arithmetic operations. As a result the cost of each arithmetic operation is quadratic in the size of the operands. Moreover, it imposes additional memory requirements for each intermediate number, which slows down the resulting computation. To reduce the memory requirements and cost of arithmetic operations, we perform our computations over finite fields and use a probabilistic algorithm based on chinese remainder theorem to recover the actual coefficients. Thus, bignum arithmetic is restricted only to the computations related to chinese remainder theorem. The complexity of the resulting algorithm is linear in the size of the coefficients of the resultant. The overall algorithm has been implemented in $\mathrm{C}++$ (as opposed to using lisp like environment) and we consider sparse as well as dense representation for multivariate polynomials (depending upon the application).

\subsection{Multivariate Interpolation}

Lets assume that each entry of the matrix is a polynomial in $x_{1}, x_{2}, \ldots, x_{n}$ and the matrix is of order $m$. If the entries are rational functions, they can be multiplied by suitable polynomials such that each entry of the resulting matrix is a polynomial. Furthermore, the coefficients of matrix entries are from a field of characteristic zero. The main idea is to determine the power products that occur in the determinant, say a multivariate polynomial $F\left(x_{1}, x_{2}, \ldots, x_{n}\right)$. Let the maximum degree of $x_{i}$ in $F\left(x_{1}, x_{2}, \ldots, x_{n}\right)$ be $d_{i}$. The $d_{i}$ 's can be determined from the matrix entries. $F$ can have at most $q_{1}=\left(d_{1}+1\right)\left(d_{2}+1\right) \ldots\left(d_{n}+1\right)$ 
monomials. In some cases, it is easier to compute a bound on the total degree of $F$. Any degree $d$ polynomial in $n$ variables can have at most $q_{2}=C(n, d)$ coefficients, where

$$
C(n, d)=\left(\begin{array}{c}
n+d-1 \\
d
\end{array}\right) .
$$

We represent the determinant as

$$
F\left(x_{1}, x_{2}, \ldots, x_{n}\right)=c_{1} m_{1}+c_{2} m_{2}+\ldots+c_{q} m_{q},
$$

where $q$ is bounded by $q_{1}$ or $q_{2}$. The $m_{i}=$ $x_{1}^{d_{1, i}} x_{2}^{d_{2, i}} \ldots x_{n}^{d_{n, i}}$ are the distinct monomials and the $c_{i}$ are the corresponding non-zero coefficients. The problem of determinant expansion corresponds to computing the $c_{i}$ 's. By choosing different substitutions for $\left(x_{1}, \ldots, x_{n}\right)$ and computing the corresponding $F\left(x_{1}, \ldots, x_{n}\right)$ (expressed as determinant of numeric matrices) the problem is reduced to that of multivariate interpolation [BT88; Zi90]. Since there are $q$ variables, we need to choose $q$ distinct substitutions and solve the resulting $q \times q$ system of linear equations. The running time of the resulting algorithm is $O\left(q^{3}\right)$ and takes $O\left(q^{2}\right)$ space. To reduce the running time and space of the algorithm we perform Vandermonde interpolation.

Let $p_{1}, p_{2}, \ldots, p_{n}$ denote distinct primes and $b_{i}=p_{1}^{d_{1, i}} p_{2}^{d_{2, i}} \ldots p_{n}^{d_{n, i}}$ denote the value of the monomial $m_{i}$ at $\left(p_{1}, p_{2}, \ldots, p_{n}\right)$. Clearly, different monomials evaluate to different values. Let $a_{i}=$ $F\left(p_{1}^{i}, p_{2}^{i}, \ldots, p_{n}^{i}\right), i=1, q . \quad a_{i}$ 's are computed by Guass elimination. Thus, the problem of computing $c_{i}$ 's is reduced to solving a Vandermonde system system $V C=A$, where

$$
\begin{gathered}
V=\left(\begin{array}{cccc}
1 & 1 & \ldots & 1 \\
b_{1} & b_{2} & \ldots & b_{k} \\
\vdots & \vdots & \vdots & \vdots \\
b_{1}^{q-1} & b_{2}^{q-1} & \ldots & b_{q}^{q-1}
\end{array}\right), \\
C=\left(\begin{array}{c}
c_{1} \\
c_{2} \\
\vdots \\
c_{q}
\end{array}\right), \quad A=\left(\begin{array}{c}
a_{1} \\
a_{2} \\
\vdots \\
a_{q}
\end{array}\right) .
\end{gathered}
$$

Computing each $a_{i}$ takes $O\left(m^{3}\right)$ time, where $m$ is the order of the matrix. As far as solving Vandermonde systems is concerned, simple algorithms of time complexity $O\left(q^{2}\right)$ and $O(q)$ space requirements are known [Zi90]. In [KL88] an improved algorithm of time complexity $O(M(q) \log (q))$ is presented, where $M(q)$ is the time complexity of multiplying two univariate polynomials of degree $q$. However, due to simplicity we have implemented the $O\left(q^{2}\right)$ algorithm and all the time complexities are computed with respect to that. Thus, the running time of the resulting algorithm is $O\left(q m^{3}+q^{2}\right)$ and space requirements are $O\left(m^{2}+q\right)$.

Before we use Vandermonde interpolation, the algorithm for computing symbolic determinant needs to know $q$ and the $m_{i}$ 's corresponding to nonzero $c_{i}$ 's. In the worst case, $q$ may correspond to $q_{1}$ or $q_{2}$ and the resulting problem is that of dense interpolation [Zi90]. The $m_{i}$ 's are enumerated in some order (e.g. lexicographic) and $b_{i}$ 's are computed by substituting $p_{j}$ for $x_{j}$. However, $q_{1}$ or $q_{2}$ tend to grow exponentially with the order of the matrix, the degree of each matrix entry and the number of variables involved. This approach becomes unattractive when the determinant is a sparse polynomial.

A sparse interpolation algorithm for such problems has been presented in [BT88]. Its time and space complexity have been improved in [KL88].

\subsubsection{Sparse Interpolation}

The algorithm in [BT88] needs an upper bound $T \geq q$ on the number of non-zero monomials in $F\left(x_{1}, \ldots, x_{n}\right)$. Given $\left(p_{1}^{j}, p_{2}^{j}, \ldots, p_{n}^{j}\right)$, it computes the monomial values $b_{i}=p_{1}^{d_{1, i}} p_{2}^{d_{2, i}} \ldots p_{n}^{d_{n, i}}$ by computing the roots of an auxiliary polynomial $G(z)$. These $b_{i}$ 's are used for defining the coefficient matrix of the Vandermonde system.

The polynomial $G(z)$ is defined as

$$
G(z)=\prod_{i=1}^{q}\left(z-b_{i}\right)=z^{k}+g_{k-1} z^{k-1}+\ldots+g_{0} .
$$

Its coefficients, $g_{i}$ 's, are computed by solving a Toeplitz system of equations [BT88]. The Toeplitz system is formed by computing the $a_{i}$ 's using the fact $G\left(b_{i}\right)=0$. Given $G(z)$, the algorithm computes its integer roots to compute the corresponding to $b_{i}$ 's. The roots are computed using p-adic root finder in [Lo83]. The running time of the resulting algorithm for polynomial interpolation is $\left(n d T^{3} \log (n)+m^{3} T\right)$. The dominating step is the polynomial root finder which takes $O\left(T^{3} \log (B)\right)$, where $B$ is an upper bound on the values of the roots.

This algorithm is unattractive for expanding symbolic determinants for the following reasons:

- It is rather difficult to come up with a sharp bound on $T$. We only have the choice of using $T=q_{1}$ or $T=q_{2}$ and the resulting problem corresponds to dense interpolation. As a result, $b_{i}$ 's can be computed directly and we do not have to use any sparse interpolation algorithm. 
- The algorithm is very slow in practice. An implementation of this algorithm using modular arithmetic is described in [KLW90]. It takes the number of variables and terms as inputs and generates a random polynomial to be used as the black box (for computing $a_{i}$ 's). The algorithm is implemented in lisp on a Sun-4. For polynomials in 3 variables and upto 250 terms this algorithm takes about 57,000 seconds. Furthermore, it almost fails to interpolate polynomials in 5 variables and having a similar bound on the number of terms.

Thus, neither the sparse nor the dense interpolation algorithms are well suited for our application.

\subsubsection{Probabilistic Interpolation}

[Zi90] presents a probabilistic algorithm for interpolation which does not expect any information about the non-zero terms of the polynomial being interpolated. It expects a bound on the degree of each variable in any monomial. Such a bound is easy to compute for a symbolic determinant. In fact, this bound is tight. The algorithm proceeds inductively, uses the degree information and develops an estimate of the number of terms as each new variable is introduced. As a result its performance is output sensitive and depends on the actual number of terms in the polynomial. We present a brief outline of the algorithm below. It has been explained in detail in [Zi90].

Choose $n$ random numbers $r_{1}, \ldots, r_{n}$ from the ring used for defining the polynomial coefficients. The algorithm proceeds inductively and introduces a variable at each stage. Let us assume we are at the $k$ th stage and have interpolated a polynomial in $k$ variables. The resulting polynomial is of the form

$$
\begin{gathered}
F_{k}\left(x_{1}, \ldots, x_{k}\right)=F\left(x_{1}, x_{2}, \ldots, x_{k}, r_{k+1}, \ldots, r_{n}\right) \\
=c_{1, k} m_{1, k}+\ldots+c_{\alpha, k} m_{\alpha, k} .
\end{gathered}
$$

In this case, $\alpha \leq q . F_{i}$ represents a polynomial in $i$ variables $x_{1}, \ldots, x_{i}$.

To

compute $F_{k+1}\left(x_{1}, \ldots, x_{k+1}\right)$ from $F_{k}\left(x_{1}, \ldots, x_{k}\right)$, it represents $F_{k+1}$ as

$$
\begin{gathered}
F_{k+1}\left(x_{1}, \ldots, x_{k+1}\right)=F\left(x_{1}, \ldots, x_{k+1}, r_{k+2}, \ldots, r_{n}\right) \\
=h_{1}\left(x_{k+1}\right) m_{i, k}+\ldots+h_{\alpha}\left(x_{k+1}\right) m_{\alpha, k},
\end{gathered}
$$

where each each $h_{i}\left(x_{k+1}\right)$ is a polynomial of degree $d_{k+1} . \quad d_{k+1}$ is a bound on the degree of $x_{k+1}$ in any term of $F\left(x_{1}, \ldots, x_{n}\right)$. It computes $h_{i}\left(x_{k+1}\right)$ by Vandermonde interpolation. The value of each $h_{i}\left(x_{k+1}\right)$ are obtained for $d_{k+1}+1$ values of $x_{k+1}$ $\left(1, p_{k+1}, p_{k+1}^{2}, \ldots, p_{k+1}^{d_{k+1}}\right)$ as follows:

$$
\begin{array}{r}
F_{k+1}\left(1, \ldots, 1, p_{k+1}^{j}, r_{k+2}, \ldots, r_{n}\right) \\
=h_{1}\left(p_{k+1}^{j}\right)+\ldots+h_{\alpha}\left(p_{k+1}^{j}\right) \\
F_{k+1}\left(p_{1}, \ldots, p_{k}, p_{k+1}^{j}, r_{k+2}, \ldots, r_{n}\right) \\
=h_{1}\left(p_{k+1}^{j}\right) b_{i, k}+\ldots+h_{\alpha}\left(p_{k+1}^{j}\right) b_{\alpha, k} \\
F_{k+1}\left(p_{1}^{2}, \ldots, p_{k}^{2}, p_{k+1}^{j}, r_{k+2}, \ldots, r_{n}\right) \\
=h_{1}\left(p_{k+1}^{j}\right) b_{i, k}^{2} \ldots h_{\alpha}\left(p_{k+1}^{j}\right) b_{\alpha, k}^{2} \\
\vdots \\
F_{k+1}\left(p_{1}^{\alpha-1}, \ldots, p_{k}^{\alpha-1}, p_{k+1}^{j}, r_{k+2}, \ldots, r_{n}\right) \\
=h_{1}\left(p_{k+1}^{j}\right) b_{i, k}^{\alpha-1}+\ldots+h_{\alpha-1}\left(p_{k+1}^{j}\right) b_{\alpha, k}^{\alpha-1}
\end{array}
$$

This is a Vandermonde system of $\alpha$ equations in $\alpha$ unknowns and can be solved for $h_{i}\left(p_{k+1}^{j}\right)$. The computation is repeated for $j=0, \ldots, d_{k+1}$. Given $h_{i}(1), h_{i}\left(p_{k+1}\right), h_{i}\left(p_{k+1}^{2}\right), \ldots, h_{i}\left(p_{k+1}^{d_{k+1}}\right)$, use Vandermonde interpolation (for univariate polynomials) to compute $h_{i}\left(x_{k+1}\right)$. These $h_{i}\left(x_{k+1}\right)$ are substituted to represent $F_{k+1}$ as a polynomial in $k+1$ variables. $F_{k+1}\left(x_{1}, \ldots, x_{k+1}\right)$ can have at most $\left(\alpha *\left(d_{k+1}+1\right)\right) \leq$ $q$ terms.

The algorithm starts with $F\left(r_{1}, \ldots, r_{n}\right)$ and computes the $n$ stages as shown above. There is a small chance that the answer produced by the algorithm is incorrect. This happens for a choice of $r_{1} \ldots, r_{n}$ and can be explained in the following manner. Let the stage $I$ of the algorithm result in a polynomial of the form

$F_{1}\left(x_{1}\right)=F\left(x_{1}, r_{2}, \ldots, r_{n}\right)=c_{0}+c_{1} x_{1}^{3}+\ldots+c_{\beta} x_{1}^{d_{1}}$.

$F_{2}\left(x_{1}, x_{2}\right)$ actually is a polynomial of the form

$$
\begin{gathered}
F_{2}\left(x_{1}, x_{2}\right)=F\left(x_{1}, x_{2}, r_{3}, \ldots, r_{n}\right) \\
=h_{1}\left(x_{2}\right)+h_{2}\left(x_{2}\right) x_{1}+h_{3}\left(x_{2}\right) x_{1}^{2}+\ldots+h_{d_{1}+1}\left(x_{2}\right) x_{1}^{d_{1}} .
\end{gathered}
$$

This implies

$h_{1}\left(r_{2}\right)=c_{0}, h_{2}\left(r_{2}\right)=0, h_{3}\left(r_{2}\right)=0, \ldots, h_{d_{1}+1}\left(r_{2}\right)=c_{\beta}$.

In practice, $h_{2}\left(x_{2}\right)$ and $h_{3}\left(x_{2}\right)$ maybe nonzero polynomials, but for the choice of $r_{2}$, where $h_{2}\left(r_{2}\right)=0$, $h_{3}\left(r_{2}\right)=0$, the algorithm assumes them as zero polynomials and proceeds. As a result the algorithm may report fewer terms in $F\left(x_{1}, \ldots, x_{n}\right)$.

Such an error is possible at each stage of the algorithm. Let us assume that $\boldsymbol{r}_{i}$ 's are chosen uniformly randomly from a set of size $S$, than the probability that this algorithm gives a wrong answer is less than

$$
\frac{n d^{2} q^{2}}{S}
$$


where $d=\max \left(d_{1}, \ldots, d_{n}\right)$ [Zi90]. This probability bound has been used to choose $r_{i}$ 's from a suitable field [MC91b]. The running time of the algorithm is $O\left(n d q^{2}+m^{3} n d q\right)$.

A deterministic version of this algorithm of complexity $O\left(n d q^{2} T+m^{3} n d q T\right)$ is given in [Zi90] as well. $T$ is an upper bound on the number of non-zero terms in the polynomial. In the worst case, $T$ corresponds to $q_{1}$ or $q_{2}$. However, the resulting algorithm has better complexity than sparse or dense interpolation algorithms. Due to simplicity and time complexity of the algorithm, we use the probabilistic version for our implementation. However, we give user the flexibility of imposing a bound on the probability of failure and choose the finite fields appropriately. Furthermore, we introduce simple, randomized checks in the algorithm to detect wrong answers

\subsubsection{Modular Arithmetic}

An important problem in the context of finite field computations is getting a tight bound on the coefficients of the resulting polynomial. Since the resultant can always be expressed as a ratio of determinants (or a single determinant), it is possible to use Hadamard's bound for determinants to compute a bound on the coefficients of the resultant [Kn81]. In practice, we found such bounds rather loose and use a randomized version of chinese remainder algorithm to compute the actual bignums. The main idea is to compute the coefficients modulo different primes, use the chinese remainder theorem for computing the bignum and make a check whether the bignum is the actual coefficient of the determinant. This process stops when the products of all the primes (used for finite field representation) is greater than twice the magnitude of the largest coefficient in the input. It is possible that for a certain choice of primes, the algorithm results in a wrong answer. The probability of failure is bounded by $l / p$, where $l$ corresponds to the number of primes used in the during the chinese remainder algorithm, $p$ is the magnitude of the smallest prime number used in this computation [MC91b].

The algorithm proceeds in stages. At the kth stage a random prime number $\left(p_{k}\right)$ is chosen and $G_{k}\left(x_{1}, \ldots, x_{n}\right)=F\left(x_{1}, \ldots, x_{n}\right) \bmod p_{k}$ is computed using the interpolation algorithm. Let

$$
G_{k}\left(x_{1}, \ldots, x_{n}\right)=c_{1, k} m_{1}+\ldots+c_{q, k} m_{q} .
$$

Thus, the coefficients of various $G_{i}$ 's satisfy the relation

$$
c_{i, 1}=c_{i} \bmod p_{1}
$$

$$
c_{i, k}=c_{i} \bmod p_{k},
$$

These $c_{i, j}$ 's are used for computing the bignum, $r_{i, k}$ using chinese remainder theorem, and satisfying the relations [Kn81]

$$
r_{i, k} \bmod p_{j}=c_{i, j}, \quad j=1, k
$$

While using chinese remainder theorem it is assumed that $r_{i, k}$ are integers lying in the range $\left(-\frac{p_{1} p_{3} \ldots p_{k}}{2}, \frac{p_{1} p_{2} \ldots p_{k}}{2}\right)$. At this stage we compare the bignums corresponding to the $(k-1)$ and $k$ stage. If

$$
r_{i, k-1}=r_{i, k}, \quad i=1, q
$$

it is reasonable to assume that $r_{i, m}=c_{i}, i=1, q$ and the algorithm terminates. The base case is $k=2$. Else we repeat the computation for the $(k+1) s t$ stage.

In general, $l+1$ prime numbers are being used, where $l$ is the minimum integer satisfying the relation

$$
2\left|c_{j}\right|<p_{1} p_{2} \ldots p_{l+1}
$$

and $\left|c_{j}\right|$ corresponds to the coefficient of maximum magnitude of the resultant and $p_{j}$ 's are randomly chosen primes. Thus, the algorithm is:

1. Compute the $c_{i, j}$ 's using the probabilistic interpolation algorithm.

2. Given $c_{i, 1}, c_{i, 2}, \ldots, c_{i, j}$, use chinese remainder theorem to compute $r_{i, j}$ for $i=1, q$.

3. If $r_{i, j-1}=r_{i, j}$ for $i=1, q$, then $c_{i}=r_{i j}$, else repeat the steps given above.

\subsection{Implementation}

We have implemented the above algorithm in $\mathrm{C}++$ on Sun-4's. The code was ported over to an IBM $\mathrm{RS} / 6000$ for performance analysis. The algorithm expects the entries of the matrix as polynomials. Given the matrix, it computes the degree bound for each variable by adding the degrees of that variable in various entries of the matrix. The total amount of space required is linear in the input size and we are easily able to run these algorithms on $8-16$ Megabyte machines.

We have implemented the dense as well as probabilistic versions of the interpolation algorithm. It can be easily interfaced with any computer algebra systems. The finite fields used for computation are of the order of $2^{30}$, on the 32 bit machines. The multiplication instruction for operands belonging to such fields has been implemented in the assembly language of the given machines.

Furthermore, it is not possible to choose any arbitrary prime for finite field computation. Let 
$V_{2}=\left(v_{2 j}\right)$ represent the elements of the second row of the Vandermonde matrix used in the interpolation algorithms. $p_{k}$ can be used as a prime for finite field computation, if and only if all the elements of the vector $V_{2 k}=\left(v_{i j}\right) \bmod p_{k}$ are distinct [MC91b].

\subsection{Applications}

We used this algorithm for implicitizing rational parametric surfaces. Given a parametrization, $(x, y, z, w)=(X(s, t), Y(s, t), Z(s, t), W(s, t))$, we formulate the parametric equations

$$
\begin{aligned}
& w X(s, t)-x W(s, t)=0 \\
& w Y(s, t)-y W(s, t)=0 \\
& w Z(s, t)-z W(s, t)=0
\end{aligned}
$$

and the problem of implicitization corresponds to computing the resultant of the above equations, by considering them as polynomials in $s$ and $t$ [MC90]. Some experiments with the implementations of Gröbner bases and resultants in Macsyma 414.62 on a Symbolics lisp machine (with $16 \mathrm{MB}$ main memory and $120 \mathrm{MB}$ virtual memory) are described in [Ho90]. For many cases of bicubic surfaces (whose highest monomial is of the form $s^{3} t^{3}$ ), these systems are unable to implicitize such surfaces and fail due to insufficient virtual memory. Only a new algorithm for basis conversion is able to implicitize such surfaces, however it takes about $10^{5}$ seconds, which would be considered impractical for most applications [Ho90].

The performance of our algorithm in the context of implicitization has been presented in Table I. Since the implicit representations are dense polynomials in general, we used dense interpolation algorithms [MC90]. The timings correspond to a single iteration over a finite field and typically $3-4$ iterations are required. As a result, it is possible to implicitize bicubic surfaces, on machines like IBM RS/6000, in less than two minutes.

\begin{tabular}{|c|c|c|c|c|}
\hline Paramet. & Degree & Terms & Sun-4 & IBM \\
\hline$s^{2}+t^{2}$ & 4 & 10 & $1 \mathrm{sec}$. & $1 \mathrm{sec}$. \\
\hline$s^{3}+t^{3}$ & 9 & 220 & $6 \mathrm{sec}$. & $3 \mathrm{sec}$. \\
\hline$s^{2} t^{2}$ & 8 & 165 & $4 \mathrm{sec}$. & $2 \mathrm{sec}$. \\
\hline$s^{3} t^{3}$ & 18 & 1330 & $100 \mathrm{sec}$. & $23 \mathrm{sec}$. \\
\hline$s^{3} t^{4}$ & 24 & 2925 & $430 \mathrm{sec}$. & $118 \mathrm{sec}$. \\
\hline
\end{tabular}

Table 1: The performance of resultant algorithm for implicitization (a single iteration over a finite field)

The algorithm has also been used for expanding symbolic determinants. More details on its performance have been given in [MC91b].

\section{Numeric Solutions}

In this section we present an algorithm to compute the numerical solutions of a given system of polynomial equations. However we are only interested in solutions lying in a subset of the real domain.

Given $n$ homogeneous equations in $n+1$ unknowns, $F_{1}\left(x_{0}, x_{1}, \ldots, x_{n}\right), \ldots, F_{n}\left(x_{0}, x_{1}, \ldots, x_{n}\right)$, where the domain of variables is limited to $1 \times$ $\left[a_{1}, b_{1}\right] \times\left[a_{2}, b_{2}\right] \times \ldots \times\left[a_{n}, b_{n}\right], x_{0}$ is the homogenizing variable and we are only interested in the affine solutions. Let

$$
F_{0}\left(x_{0}, x_{1}, \ldots, x_{n}\right)=u_{0} x_{0}+u_{1} x_{1}+\ldots+u_{n} x_{n},
$$

be a linear polynomial and $R\left(u_{0}, u_{1}, \ldots, u_{n}\right)$ be the resultant of $F_{0}, F_{1}, \ldots, F_{n}$ obtained by considering them as polynomials in $x_{0}, x_{1}, \ldots, x_{n}$. It is a homogeneous polynomial in $u_{i}$ 's and its degree is equal to the product of the degrees of $F_{i}$ 's. $R\left(u_{0}, \ldots, u_{n}\right)$ is the u-resultant of the given system of equations and factors into linear factors of the form

$$
\alpha_{0} u_{0}+\alpha_{1} u_{1}+\ldots+\alpha_{n} u_{n}
$$

where $\left(\alpha_{0}, \alpha_{1}, \ldots, \alpha_{n}\right)$ correspond to the solution of the original system [Wd50]. However, computing the expression $R\left(u_{0}, \ldots, u_{n}\right)$ and factoring into linear factors can be a time consuming task, even for low degree polynomials. We therefore, specialize some of the $u_{i}$ 's and reduce the problem to computing roots of univariate polynomials in a real interval. For many small values of $n$ and certain combinations of the degrees of $F_{i}$ 's, the resultant can be expressed as determinant of a matrix. Otherwise the resultant can be expressed as a ratio of two determinants and in either case the entries of the matrices are polynomials in $u_{0}, u_{1}, \ldots, u_{n}$. After specialization, these entries are univariate polynomials in $u_{0}$ and the problem of computing roots of the univariate polynomial corresponding to the determinant (or ratio of determinants) can be reduced to an eigenvalue problem. Efficient algorithms for computing the eigenvalues of matrices are given in [GV89] and good implementations are available as part of standard packages like EISPACK and LAPACK [De89].

We assume that the original system has no solutions at infinity. To circumvent this problem we can apply a generic non-singular linear transform to the coordinates, $\left(x_{0}, x_{1}, \ldots, x_{n}\right)$, and appropriately adjust the domain of the modified system of equations.

Let

$$
f_{1}\left(u_{0}\right)=R\left(u_{0}, u_{1}=1, u_{2}=0, \ldots, u_{n}=0\right)
$$

be a polynomial of degree $d$. Since it corresponds to a projection of the $u$-resultant, it can be factored into 
linear factors of the form

$$
k u_{0}^{m}\left(u_{0}+\alpha_{11}\right)\left(u_{0}+\alpha_{12}\right) \ldots\left(u_{0}+\alpha_{1 q}\right),
$$

where each $\alpha_{1 i}$ corresponds to the projection on the $x_{1}$ coordinate. We are only interested in roots lying in the interval $\left[a_{1}, b_{1}\right]$. Later on we reduce this problem to computing eigenvalues of matrices. Let there be $p_{1}$ such roots, $L_{1}=\left(\alpha_{1 j}, \ldots, \alpha_{1 p_{1}}\right)$.

Similarly we compute the roots of

$$
f_{2}\left(u_{0}\right)=R\left(u_{0}, u_{1}=0, u 2=1, u_{3}=0, \ldots, u_{n}=0\right)
$$

in the interval $\left[a_{2}, b_{2}\right]$, say $L_{2}=\left(\alpha_{21}, \alpha_{22}, \ldots, \alpha_{2 p_{2}}\right)$. Since two projections are not enough for establishing the correspondence between the projections on $u_{1}$ and $u_{2}$ coordinates, we take a generic combinations of these two coordinates and let

$f_{1,2}\left(u_{0}\right)=R\left(u_{0}, u_{1}=k_{1}, u_{2}=k_{2}, u_{3}=0, \ldots, u_{n}=0\right)$,

where $k_{1}$ and $k_{2}$ are two positive random numbers. Let $L_{1,2}=\left(\beta_{1}, \beta_{2}, \ldots, \beta_{p_{1,2}}\right)$ be its roots in the interval $\left[k_{1} a_{1}+k_{2} a_{2}, k_{1} b_{1}+k_{2} b_{2}\right]$. To establish the correspondence between the projections on $u_{1}$ and $u_{2}$ of the actual roots, we compute all the combinations of the form $k_{1} \alpha_{1}+k_{2} \alpha_{2}$, where $\alpha_{1} \in L_{1}$ and $\alpha_{2} \in L_{2}$ and compare them with the elements in $L_{1,2}$. Since our projection on $u_{1}$ and $u_{2}$ is a generic projection, it is reasonable to assume that the exact matches correspond to the projections of the roots of $F_{i}$ 's on $x_{1}$ and $x_{2}$ coordinates. In a similar manner, we compute the roots of $f_{3}\left(u_{0}\right), f_{2,3}\left(u_{0}\right), \ldots, f_{n}\left(u_{0}\right), f_{n-1, n}\left(u_{0}\right)$ in the corresponding intervals, where

$$
\begin{aligned}
& f_{i}\left(u_{0}\right)=R\left(u_{0}, u_{1}=0, \ldots, u_{i-1}=0, u_{i}=1\right. \text {, } \\
& u_{i+1}=0, \ldots, u_{n}=0 \text { ) } \\
& f_{i, j}\left(u_{0}\right)=R\left(u_{0}, u_{1}=0, \ldots, u_{i-1}=0, u_{i}=k_{i}, \ldots,\right. \\
& u_{j-1}=0, u_{j}=k_{j}, u_{j+1}=0, \ldots, u_{n}=0 \text { ) }
\end{aligned}
$$

$k_{i}$ and $k_{j}$ are random positive integers. These roots can be used to compute the rest of the $x_{i}$ coordinates of the solutions of the original system of equations. It is possible that the resulting solution set contains some extraneous solutions. As a result, we back substitute the roots in the original system of equations to eliminate the extraneous roots from the solution set. More details on the algorithm are presented in [MC91b].

\subsection{Reduction to Eigenvalue Problem}

In the previous section we reduced the problem of computing solutions of a system of multivariate polynomials (in the domain of interest) to finding roots of univariate polynomials in suitable intervals. The univariate polynomials, like $f_{i}\left(u_{0}\right)$, are expressed as a determinant or as a ratio of two determinants and we are interested in roots lying in the interval $[a, b]$. Let us consider the case when it is expressed as a ratio of two determinants and the corresponding matrices are denoted as $M\left(u_{0}\right)$ and $D\left(u_{0}\right)$. In case, the resultant corresponds to a determinant of a matrix, Determinant $\left(D\left(u_{0}\right)\right)=1$. Each entry of $M\left(u_{0}\right)$ and $D\left(u_{0}\right)$ is a polynomial in $u_{0}$. Let its degree be bounded by $d$. Depending upon the value of $D\left(u_{0}\right)$ there are two possible cases:

- Determinant $\left(D\left(u_{0}\right)\right) \neq 0$. Thus,

$$
f_{i}\left(u_{0}\right)=\frac{\operatorname{Determinant}\left(M\left(u_{0}\right)\right)}{\operatorname{Determinant}\left(D\left(u_{0}\right)\right)}
$$

Let $S_{1}$ and $S_{2}$ be the solution sets corresponding to the roots of $\operatorname{Determinant}\left(M\left(u_{0}\right)\right)=0$ and Determinant $\left(D\left(u_{0}\right)\right)=0$ lying in the interval $[a, b]$, respectively. As a result, the roots of $f_{i}\left(u_{0}\right)$ correspond to $S_{1} \backslash S_{2}$. We reduce the problem of computing $S_{1}$ or $S_{2}$ to an eigenvalue problem.

- Determinant $\left(D\left(u_{0}\right)\right)=0 . \quad$ As a result Determinant $\left(M\left(u_{0}\right)\right)=0$. We consider a nonvanishing minor of $M\left(u_{0}\right)$, say $M_{1}\left(u_{0}\right)$ (of maximum ranks among all such minors). All the roots of $f_{i}\left(u_{0}\right)$ are contained in the roots of Determinant $\left(M_{1}\left(u_{0}\right)\right)$. We compute these roots by reducing it to an eigenvalue problem.

Let us assume that $M\left(u_{0}\right)$ is a matrix of order $n$. Each entry of $M\left(u_{0}\right)$ is a polynomial of degree $d$ and it can therefore, be represented as

$$
M\left(u_{0}\right)=u_{0}^{d} M_{d}+u_{0}^{d-1} M_{d-1}+\ldots+u_{0} M_{1}+M_{0},
$$

where $M_{i}$ 's are matrices of order $n$ with numeric entries. Let us assume that $M_{d}$ is a non-singular matrix. As a result, the roots of the following equations are equivalent

$$
\operatorname{Determinant}\left(M\left(u_{0}\right)\right)=0
$$

$$
\operatorname{Determinant}\left(M_{d}^{-1}\right) \operatorname{Det}\left(M\left(u_{0}\right)\right)=0 \text {. }
$$

Let

$$
\bar{M}\left(u_{0}\right)=u_{0}^{d} I_{n}+u_{0}^{d-1} \bar{M}_{d-1}+\ldots+u_{0} \bar{M}_{1}+\bar{M}_{0}
$$

where

$$
\bar{M}_{i}=M_{d}^{-1} M_{i}, \quad 0 \leq i<d
$$

and $I_{n}$ is an $n \times n$ identity matrix. Given $\bar{M}\left(u_{0}\right)$, we use Theorem 1.1 [GLR82] to construct a matrix 
of the form

$$
C=\left[\begin{array}{ccccc}
0 & I_{n} & 0 & \ldots & 0 \\
0 & 0 & I_{n} & \ldots & 0 \\
\vdots & \vdots & \vdots & \vdots & \vdots \\
0 & 0 & 0 & \ldots & I_{n} \\
-\bar{M}_{0} & -\bar{M}_{1} & \bar{M}_{2} & \ldots & \bar{M}_{d-1}
\end{array}\right]
$$

such that the eigenvalues of $C$ correspond exactly to the roots of $\operatorname{Det}(\bar{M}(u))=0$. C is a numeric matrix of order $d n$. If $M_{d}$ is a singular matrix, techniques to compute the roots of $\operatorname{Det}(M(u))=0$ are given in [GLR82].

\subsubsection{Implementation}

We used EISPACK routines for computing the eigenvalues of matrices. Many special purpose algorithms are available for computing the eigenvalues of matrices, which make use of the structure of the matrix. As far as matrix $C$ in (3) is concerned, we treat it as a general unsymmetric matrix. We used the routine RG from EISPACK for computing the eigenvalues [GBDM77]. Given a general unsymmetric matrix, it makes use of balancing techniques, reduces it to upper Hessenberg form and uses the shifted QR algorithm on the resulting matrix to compute the eigenvalues [GV89]. The current implementation of these routines compute all the eigenvalues. The performance of eigenvalue computation routines for matrices of different order (generated randomly) are given in Table II. The timings correspond to the implementation on an IBM RS/6000.

\begin{tabular}{|c|c|}
\hline Order of Matrix & Time in seconds \\
\hline 15 & $8631.839844 \times 10^{-6}$ \\
\hline 20 & $15717.63965 \times 10^{-6}$ \\
\hline 25 & $25753.00000 \times 10^{-6}$ \\
\hline 30 & $38763.23828 \times 10^{-6}$ \\
\hline 35 & $57124.16016 \times 10^{-6}$ \\
\hline 40 & $77398.03906 \times 10^{-6}$ \\
\hline 45 & $103343.5234 \times 10^{-6}$ \\
\hline 50 & $133956.2344 \times 10^{-6}$ \\
\hline 55 & $165395.0469 \times 10^{-6}$ \\
\hline 60 & $212041.2812 \times 10^{-6}$ \\
\hline 65 & $262103.1250 \times 10^{-6}$ \\
\hline
\end{tabular}

Table II

The performance of eigenvalue computation routines

The timings given in Table II are satisfactory for most applications. We are currently working on modifying the algorithms to compute the eigenvalues lying in an interval like $[a, b]$ and as a result, expect a better performance.

\section{Conclusion}

In this paper we have presented algorithms to efficiently compute the resultants of polynomial equations and using properties of matrices and determinants used them to compute the roots of a system of polynomial equations. As a result, it is possible to perform symbolic elimination from a given set of polynomial equations in a reasonable amount of time and space requirements. We have used these algorithms for implicitizing parametric surfaces, problems in inverse kinematics and computing the configuration space for curved objects for robot motion planning.

\section{Acknowledgements}

We are grateful to Prof. J. Demmel and Prof. W. Kahan for productive discussions. This research was supported in part by David and Lucile Packard Fellowship and National Science Foundation Presidential Young Investigator Award (\# IRI-8958577).

\section{References}

[BGW88] Bajaj, C., Garrity, T. and Warren, J. (November 1988) "On the applications of multi-equational resultants", Tech. report CSD-TR-826, Computer Science Deptt., Purdue University.

[Bu85] Buchberger, B. (1987) "Gröbner bases: An algorithmic method in polynomial ideal theory", in Multidimensional Systems Theory, edited by N.K. Bose, pp. 184-232, D. Reidel Publishing Co..

[Bu89] Buchberger, B. (1989) "Applications of Grőbner bases in non-Linear computational geometry", in Geometric Reasoning, eds. D. Kapur and J. Mundy, pp. 415-447, MIT Press.

[BT88] Ben-Or, M. and Tiwari, P. (1988) "A deterministic algorithm for sparse multivariate polynomial interpolation", 20th Annual ACM Symp. Theory of Comp., pp. 301-309.

[Ca87] Canny, J. F. (1987) The complexity of robot motion planning, ACM Doctoral Dissertation award, MIT Press.

[Ca90] Canny, J. F. (1990) "Generalized characteristic polynomials", Journal of Symbolic Computation, vol. 9, pp. 241-250.

[De89] Demmel, J. (1989) "LAPACK: A portable linear algebra library for supercomputers", IEEE Control systems society workshop on computer-aided control system design, Tampa, Florida.

[Di08] Dixon, A.L. (1908) "The eliminant of three quantics in two independent variables", Proceedings of London Mathematical Society, vol. 6, pp. 49-69, 
473-492.

[GLR82] Gohberg, I., Lancaster, P. and Rodman, L. (1982) Matrix polynomials, Academic Press, New York.

[GV89] Golub, G.H. and Van Loan, C. F. (1989) Matrix computations, The John Hopkins Press, Baltimore, Maryland.

[Ho90] Hoffmann, C. (1990) "Algebraic and numeric techniques for offsets and blends", in Computation of Curves and Surfaces, eds. W. Dahmen et. al., pp. 499-529, Kluwer Academic Publishers.

[Jo89] Jouanolou, Jean-Pierre (1989) "Le Formalisme Du Résultant", Department of Mathemetics, Université Louis Pasteur, France.

[KL88] Kaltofen, E. and Lakshman, Y.N. (1988) "Improved sparse multivariate polynomial interpolation algorithms", in Lecture Notes in Computer Science, vol. 358, pp. 467-474, Springer-Verlag.

[KLW90] Kaltofen, E., Lakshman, Y.N. and Wiley, J. (1990) "Modular rational sparse multivariate polynomial interpolation", in Proceedings of IS$S A C^{\prime} 90$ pp. 135-140, Addison-Wesley, Reading, Massachusets.

[Kn81] Knuth D. (1981) The Art of Computer Programming, Vol II , Seminumerical Algorithms Addison-Wesley . (

) [Lo83] Loos, R. 1983 "Computing rational zeros of integral polynomials by p-adic expansion",.

SIAM Journal on Computing, vol. 7, pp. 286-293 [Ma2] Macaulay, F. S. (May 1902) "On some formula in elimination", Proceedings of London Mathematical Society, pp. 3-27.

[Ma21] Macaulay, F. S. (June 1921) "Note on the resultant of a number of polynomials of the same degree", Proceedings of London Mathematical Society, pp. 14-21.

[MC27] Morley, F. and Coble, A.B. (1927) "New results in elimination", American Journal of Mathematics, vol. 49, pp. 463-488.

[MC90] Manocha, D. and Canny, J. (1990) "Algorithms for implicitizing rational parametric surfaces", to appear in Proceedings of IVIMA Conference on Mathematics of Surfaces, Claredon Press, Oxford. Also available as Tech. report UCB/CSD 90/592, Computer Science Division, Univerisity of California, Berkeley.

[MC91a] Manocha, D. and Canny, J. (1991) "A new approach for surface intersection", in Proceedings of First ACM Symposium on Solid Modeling Foundations and CAD/CAM Applications. Also available as RAMP memo. 90-11/ERSC 90-23, Engineering System Research Center, University of California, Berkeley.

[MC91b] Manocha, D. and Canny, J. (1991) "Multipolynomial Resultant Algorithms", Technical Re- port, Computer Science Division, University of California, Berkeley.

[Mi90] Milne, P. (1990) "On the solutions of a set of polynomial equations", manuscript, Department of Computer Science, University of Bath, England.

[MM82] Mayr E. and Meyer A. (1982) "The complexity of the word problem in commutative semigroups and polynomial ideals", Advances in Mathematics, vol. 46, pp. 305-329.

[Mo25] Morley, F. (1925) "The eliminant of a net of curves", American Journal of Mathematics, vol. 47, pp. 91-97.

[Mr87] Morgan, A.P. (1987) Solving polynomial systems using continuation for scientific and engineering problems, Prentice-Hall, Englewood Cliffs, New Jersey.

[Mr90] Morgan, A.P. (1990) "Polynomial continuation and its relationship to the symbolic reduction of polynomial systems", presented at the workshop on Integration of Numeric and Symbolic Computing Methods, Saratoga Springs, New York.

[Sa1885] Salmon, G. (1885) Lessons introductory to the modern higher algebra, G.E. Stechert \& Co., New York.

[Wd50] van der Waerden B. L. (1950) Modern algebra, (third edition) F. Ungar Publishing Co., New York. [WM90] Wampler, C. and Morgan, A. (1990) "Numerical continuation methods for solving polynomial systems arising in kinematics", ASME Journal on Design, vol. 112, pp. 59-68.

[Zi90] Zippel, R. (1990) "Interpolating polynomials from their values", Journal of Symbolic Computation, vol. 9, 375-403. 